\title{
Socioecological factors' effect on the subjective experience of COVID-19 quarantine policy in Korea: Consensual qualitative research
}

\section{Gee Yeon Ro}

Seoul National University

Yun-Jeong Shin ( $\nabla$ yj.shin@snu.ac.kr)

Seoul National University https://orcid.org/0000-0002-1236-1781

\section{Sunghyuck Mah}

Seoul National University

Yeseul Min

Seoul National University

Hyunjung Park

Seoul National University

Sodam Jeong

Seoul National University

\section{Research}

Keywords: COVID-19, quarantine, subjective experience, ecological influence, CQR

Posted Date: April 9th, 2021

DOl: https://doi.org/10.21203/rs.3.rs-400738/v1

License: (c) (i) This work is licensed under a Creative Commons Attribution 4.0 International License.

Read Full License 


\section{Abstract}

Background: In face of the COVID-19, South Korea has provided the 'Global Golden Standard' of containment effort. Mandatory quarantine, one of the core policies in place, has proven its efficacy in ensuring public health. Nonetheless, no previous study has examined the policy's comprehensive impact on its subjects. In addition to providing an account of holistic subjective experience of quarantine, this study also examines the socioecological factors' influence on the subjective experience by applying the Bronfenbrenner's model. In order to gather unconstrained information in relation to the contextual background, Consensual Qualitative Research method was used. 17 adults of Korean nationality were interviewed about their experience.

Results: 10 categories within four domains of Subjective experience were found: (a) changed life style because of quarantine, continued pre-quarantine life, lasting effect of quarantine in Lifestyle domain; (b) Physical health domain with no subcategory; (c) discomfort, infection anxiety, accepting, satisfying, and gratitude within Psychological Experience domain; and (d) suggestion and change of perspective within Reflection domain. Next, 13 categories of socioecological factors belonged to four different levels of domain: (a) personality and belief in Within-individual domain; (b) quarantine space, personal relationship, coresident, student status, and employment status in Microsystem; (c) Korea's quarantine policy, maintenance, resources, and abroad's preventive measure against COVID in Exosystem; and (d) stigma and social responsibility in Macrosystem.

Conclusions: The reported subjective experiences of self-quarantine were not uniformly negative or positive, which is unlike previous research findings that were dominantly negative. Identifying socioecological factors that shape an individual's quarantine experience shed light onto how the government can protect its people from the potential threats of quarantine. The examples include promoting sense of safety through clear and coherent communication about the disease and the measures that are being placed, maximizing opportunities for the subjects to exert control over their lives during quarantine, devising ways to make virtual social connection accessible, and etc.

\section{Background}

COVID-19 has been one of the biggest and most widespread pandemics of human history. While it was first identified in Wuhan, China in December 2019, it soon became a worldwide concern due to its highly contagious nature. On January $30^{\text {th }}$ of 2020 , World Health Organization (WHO) declared it to be a Public Health Emergency of International Concern [1]. The uncontrollable spread of the disease has caused enormously serious harm to the medical, social, and economic ecosystems of the world. Consequently, governments have implemented various policies to minimize further calamities.

While the policies around the world are vastly different, South Korea has provided the 'Global Golden Standard' in responding to COVID [2]. While many aspects of Korean response to COVID contributed to this unique success, mandatory quarantine is more noteworthy than others. This is because detection of 
the infected and prevention of contact between the infected and the noninfected are essential in successful management of the disease, as COVID is transmitted through human contact.

Currently in Korea, anyone who may have been in contact with infected individuals, and those entering from abroad must quarantine for two weeks. Of course, those traveling for national or public interest are exempt from this duty. Korean Center for Disease Control (KCDC) set various rules that the subjects need to abide by for 14 days [3]. They cannot make any direct or indirect (sharing food or objects) contact with another person, and must maintain personal sanitation. Furthermore, they must download the 'Safe Quarantine Application' on their phones and record their temperatures into the app twice a day. Lastly, when government workers make their daily contacts, they must report any potential symptoms of COVID. If one resists these rules, they must wear location-tracking bracelets [4].

While quarantine has proven effective in promoting public health [5], no previous study has closely examined the policy's comprehensive impact on its subjects. Prior studies have each reported on quarantine's negative impact on the subjects' psychological, behavioral, physical health. Psychologically, subjects experience fear about contracting COVID, feeling of being stigmatized, and etc [6]. Furthermore, quarantined youths experience greater psychological distress, such as worry, helplessness, and fear, when compared to non-quarantined groups [7]. Quarantine, usually characterized by increase in consumption of alcohol and food with low nutrition [8], can have long-term effects on cardiovascular disease [9]. Since it is clear that quarantine can have permeating effect on an individual's overall health, it is necessary to explore its holistic impact.

While observing the subjective experience itself does bring insight to the quarantine experience, the individuals' experiences are always under the influence of relevant ecological factors. For example, external support such as family, friends, health professionals, and a comfortable living environment can alleviate the negative mood that arise during quarantine [6]. Unfortunately, while researchers so far were able to touch upon the interplay between ecological factors and one's experience, their scope was limited to the 'external support to cope with emotional changes during quarantine' [6]. However, external factors that impact an individual's experience can exist in a non-supportive context too, and quarantine can bring more than emotional changes. Thus, the current study will thoroughly dissect the influences of various systems that surround an individual in addition to delineating the individual's experiences.

Bronfenbrenner's socioecological model will be applied to analyze the exchange between the socioecological factors and the subjective experience [10]. According to Bronfenbrenner, an individual continuously influences, and is influenced by, the following systems [10]. Microsystem refers to one's most immediate environment such as family, school, and friends. Mesosystem is the interaction between multiple microsystems, such as interaction between an individual's parents and friends. Exosystem is a system that indirectly influences an individual; media, public policies, and etc. Macrosystem refers to attitudes, ideologies, and values of the culture that an individual is part of. Lastly, Chronosystem is the changes that occur over time that influence development, such as marriage, divorce, retirement, and etc. For the purpose of this research, only the influences of one's microsystem, exosystem, and macrosystem 
will be analyzed, as the rest are polydimensional. Mesosystem requires an interaction, and chronosystem is inclusive of all other systems. This polydimensional nature could hinder the analysis of a system's unique influence over an individual.

For the purpose of this study, Consensual Qualitative Research (CQR) was utilized [11]. CQR is the most fitting research method for this study for two reasons. CQR allows the researchers to gather unconstrained responses of participants [11]. This trait is necessary to capture the comprehensive experience of the participants. Secondly, CQR provides understanding of the participants' experiences in relation to the context through inductive and rigorous analysis, and the infusion of multiple viewpoints through consensus building [11-13]. This trait is crucial to extract the contextual influence of various systems on the subjective experience. Thus, by using the CQR, we examined the holistic experience of quarantine by asking the following two questions: (a) What is the subjective experience of quarantine like? and (b) What ecological factors influence the subjective experience of quarantine?

\section{Methods}

\section{Participants}

We recruited 17 participants (four males and 13 females) ranging in age from 20 to 47 ( $M=29.88, S D=$ 6.83). 13 participants were students, and four were working professionals. 15 participants experienced self-quarantine due to their entrance from another country, and two did so because they shared space with a COVID-19 patient.12 out of 17 participants had co-residents in the quarantine space. 13 participants engaged in mandatory quarantine while four participants quarantined voluntarily (Table 1).

Table 1 Participant characteristics 


\begin{tabular}{|llll|}
\hline Characteristics & & N & $\%$ \\
\hline Age & $20-29$ & 11 & $64.7 \%$ \\
& $30-39$ & 4 & $23.5 \%$ \\
\hline Gender & $\mathbf{4 0 - 4 9}$ & $\mathbf{2}$ & $\mathbf{1 1 . 8 \%}$ \\
& Female & 13 & $76.5 \%$ \\
\hline Occupation & Male & 4 & $23.5 \%$ \\
\hline Reason for quarantine & Student & 14 & $82.4 \%$ \\
& Working professional & 3 & $17.6 \%$ \\
\hline Coresident in quarantine space & Entered Korea from another country & 15 & $88.2 \%$ \\
& Yndirect contact with a COVID-19 patient & 2 & $11.8 \%$ \\
\hline Type of quarantine & No & 12 & $70.6 \%$ \\
& Obligatory & 5 & $29.4 \%$ \\
\hline & Voluntary & 13 & $76.5 \%$ \\
\hline
\end{tabular}

\section{Research Team}

As Hill et al. recommended, we created a research team consisting of five graduate students - four doctoral students and one master student - with training experience in CQR, and one auditor who is a professor and licensed psychologist with extensive experience with conducting CQR research [11]. All researchers are pursuing counseling psychology, except for the one doctoral student who is majoring in organizational psychology in the business department. As a research team, we have experience working in agency, school, and hospital settings.

\section{Recruitment}

After getting approval from the Institutional Review Board (IRB) at authors' affiliation, we distributed recruitment advertisements to bloggers who shared that they were in quarantine, and used snowball sampling through personal contacts. Those who were interested in participating contacted the researchers. Once connected, researchers explained the purpose and procedures of the study, and screened their eligibility to participate. The inclusion criteria were as follows: (1) experienced selfquarantine due to entrance from abroad or close contacts with COVID-19 patients; (2) voluntarily participated in the study; and (3) a Korean citizen over 18 years old. The exclusion criteria were: (1) did not complete self-quarantine or (2) unable to communicate effectively. Among 18 potential participants, one was excluded from the final participant pool due to unresponsiveness. 


\section{Interviewing and transcription}

Once deemed eligible, participants gave their consents by signing the consent form. We then conducted semi-structured, one-on-one, in-depth interviews that lasted around 45-80 minute via ZOOM or in person. We strictly adhered to the sanitary guidelines to prevent COVID-19 in case of in-person interviews. The interview environments were quiet, and interruptions were minimal. We created an interview protocol that we designed (Appendix A). With the permission of the participants, we audio recorded the interview to boost the accuracy of transcription. The interviews were transcribed and assigned a code number.

\section{Procedures for Data Analysis}

Researchers engaged in continuous data analysis and frequent discussions with each other to identify the themes and subthemes. First, we individually read the transcripts for emergent domains - topic areas that accurately capture the participants' experiences -, and converted the raw data into core ideas [11, 14]. We then cross-analyzed the representativeness of domains and core ideas. Following the initial analysis, the auditor reviewed the work to ensure that the core ideas fit well into the domains, and to confirm that the labels accurately capture the nature of the categories. Using the auditor's feedback, the team modified the list of domain and core ideas once a consensus was reached that they are still reflective of the raw data. Finally, we coded the raw data into corresponding core ideas and assigned frequency labels.

\section{Results}

As a result of analyzing the data to investigate the subjective experience of the participants' selfquarantine, four domains were found; Lifestyle, Physical health, Psychological experience, and Reflection. Next, exploring the multiple layers of contextual influences on the subjective experiences of quarantine in South Korea revealed four domains with 13 categories, (a) personality and belief in Within-individual domain, (b) quarantine space, personal relationship, coresident, student status, and employment status in Microsystem, (c) Korea's quarantine policy, maintenance, resources, and abroad's preventive measure against COVID in Exosystem, and (d) stigma and social responsibility in Macrosystem. Frequency labels, General, Typical, and Variant, are based on a total of 17 cases. General describes 16 or 17 cases, Typical describes 9 to 15 cases, and Variant describes 8 or less cases.

\section{Subjective Experience of Self-Quarantine}

The consensus meaning of four domains of Korean participants' subjective experience of quarantine is as follows. Lifestyle refers to the activities and behaviors that the participants engaged in as a result of, and during, quarantine. Physical health refers to the phenomenon that manifested itself in the physical body as a result of, and during, quarantine. Psychological experience encompasses various internal experiences that arose within the participants, and Reflection presents the participants' afterthoughts of quarantine (Table 2).

Table 2 Subjective Experience 


\begin{tabular}{|lll|}
\hline Domain & Category & Frequency \\
\hline Lifestyle & Changed lifestyle because of quarantine & $11 /$ Typical \\
\hline & Continued pre-quarantine life & $16 /$ General \\
\hline Physical health & Lasting effect of quarantine & $5 /$ Variant \\
\hline Psychological experience & Discomfort & $10 /$ Typical \\
\hline & Infection Anxiety & $13 /$ Typical \\
\hline & Accepting & $6 /$ Variant \\
\hline & Satisfying & $14 /$ Typical \\
\hline Geflection & Gratitude & $6 /$ Variant \\
\hline & Suggestion & $8 /$ Typical \\
\hline & Change of Perspective & $7 /$ Variant \\
\hline
\end{tabular}

\section{Lifestyle}

The domain Lifestyle consisted of three different core ideas: changed lifestyle because of quarantine, continued pre-quarantine life, lasting effect of quarantine. Of the 17 participants, 11 reported that strictly following the quarantine regulations typically changed their lifestyles. The more strictly a participant followed the rules, the greater was the change in one's lifestyle. One participant used the kitchen only in the middle of the night, when "no one else would be awake", to minimize contact with the family members. Another participant had to "hand-wash the laundry every morning" to not use the same washing machine as the family.

On the other hand, not all participants experienced changes in lifestyle due to quarantine. In fact, participants generally continued their pre-quarantine lives without detecting much quarantine-specific differences. They continued their schoolwork and work through online mediums. Some participants continued their pre-quarantine lives, but with more emphasis on recharging over responsibility. For example, they "rested and got enough sleep", "focused on my hobby like making Gundam figures and puzzles. Time flies when I play with these things. So I just did those things freely without much concern".

Regardless of the degree of changes in lifestyle, quarantine had variantly lasting effects on some participants' post-quarantine lives. Those who felt unprecedentedly constrained and trapped during quarantine had a harder time staying indoors than ever before. These participants felt that they "cannot stand being at home more than before", and were "sick and tired of being home". They would rather be outside with a mask than stay inside without one, and tried to stay indoors as little as possible. Some participants even wanted to distance themselves from their quarantine spaces. 
On the other hand, quarantine left opposite lasting effects on others. These participants reported that they became more comfortable with staying at home as they adapted to their quarantine experience. They felt it was safer to stay at home due to the still-standing COVID-19 situation. "Even though the quarantine was over, I decided not to go outside because I felt okay and safer to be at home". Some participants grew a tendency to stay inside because they felt that keeping their masks on outside felt just as stuffy. "In Korea, I have to wear a mask when I go outside. But when I wear it, it makes me stuffy and out of breath. So, l'd rather not go out".

\section{Physical Health}

The second domain of subjective experience of quarantine was Physical health. Participants typically reported that quarantine had physical consequences. Concerns surrounding actual or the potential of weight gain were the most prevalent. Due to quarantine, they could not engage in much physical activity. On the contrary, their food intake remained the same or increased. These factors together resulted in concerns of weight gain. Furthermore, few participants shared that quarantine took quite a toll on the body. They struggled from jet lag, which made them "fall asleep when I shouldn't, and stay awake at night. Not having enough sleep made my mental state worse".

\section{Psychological Experience}

Alongside Lifestyle and Physical health, Psychological experience was the third domain, and could be categorized into five categories: discomfort, infection anxiety, accepting, satisfying, gratitude. First, participants typically identified discomfort as the challenging psychological experience of quarantine. Reports of feeling constrained were the most common, while the type and intensity of discomfort that participants experienced ranged from mere boredom to depressive symptoms. Few were tempted to "go outside...I felt healthy and my COVID screening result was negative". Some also "felt completely alone. And I could see that feeling of solitude intensifying into depression".

Participants variantly reported infection anxiety. All participants were obligated to quarantine because the possibility of becoming a COVID patient could not be ruled out. This possibility triggered infection anxiety, as they started to see normal physical phenomena such as "coughing here and there" as potential COVID symptoms. "I heard on the news that some people tested positive on their second test even if they already tested negative on their first one. So, I was anxious that my result was a false negative". Unfortunately, infection anxiety remained high until they "got a negative at that second screening".

Nonetheless, not all psychological experiences were negative. Typically, participants accepted the situation as a necessary public health policy instead of as an unnecessary enforcement. One participant shared, "I said earlier that I felt shunned when I saw my mom disinfecting the stuff I touched. But after one day, I started to think, 'Of course she should'. I got used to it quickly, and my parents and I prioritized COVID prevention". Some participants "controlled emotions by changing my thoughts. If you think being in quarantine is tough, the struggle will never diminish". 
Furthermore, participants variantly experienced satisfaction during quarantine, as they utilized the 2-week period as a very concentrated time for their needs and desires. Participants who needed to fulfill their academic responsibilities such as "TAing...papers, researches, and final" experienced quarantine as an "undisturbed time that increased my productivity and concentration". Furthermore, participants who "genuinely enjoy being home...[1] was really happy that I had to be home".

Lastly, gratitude towards the situation was typically observed among participants. These participants viewed quarantine as a tool that the government was utilizing "to contain COVID effectively" for public health promotion, and felt "especially safe" to abide by the rules. In addition, they saw quarantine as an opportunity to be "home with...mom's home cooked meals". In fact, participants typically remained in Korea even after the quarantine.

\section{Reflection}

The last domain of subjective experience of quarantine was participants' Reflection of their experiences, and mainly involved suggestions for the future and changed perspectives. First, suggestions for the future quarantine experience included "bringing some kind of entertainment to make time go by faster". Suggestions for the systematic structure that runs the quarantine policy were targeted towards the government workers. One participant stated, "They should give the workers more incentives to increase their motivation. Because COVID is here for the long run, there is always a possibility for the situation to get worse and for them to be busier. They probably already feel like their work is worthy, but giving them more practical affirmations can't hurt. A raise would be a good idea".

In addition, as quarantine inevitably created distance between the participants and their ordinary lives, they were able to view their lives with a new perspective. More specifically, they were able to realize that "routine is not boring, but is a source of calmness", and that ordinary things such as "delivery services...kitchenware....and human connection are essential to surviving". Quarantine also had the effect of expanding one's perspective towards the pandemic situation itself. One participant said "Ever since I went through quarantine, I have more understanding towards the people who unfortunately spread the disease by moving around while they were infected. I used to blame them for being selfish, but I now realize that they were just living their lives. They just didn't know that they had the virus. So now, I feel like it's not really their fault. Or actually, anyone's fault".

\section{Ecological Factors Influencing the Subjective Experience of Quarantine}

Bronfenbrenner's socioecological model was used to examine the effects of the various layers of one's surrounding on subjective experiences of self-quarantine. Four domains of ecological factors were found: Within-individual, Microsystem, Exosystem, and Macrosystem (Table 3).

Table 3 Ecological factors that affect subjective experience of quarantine 


\begin{tabular}{|lll|}
\hline Domain & Category & Frequency \\
\hline Within-Individual & Personality & $14 /$ Typical \\
\hline Microsystem & Belief & $10 /$ Typical \\
\hline & Quarantine space & $9 /$ Typical \\
\hline & Personal Relationships & $12 /$ Typical \\
\hline Exosystem & Soresident & $10 /$ Typical \\
\hline & Korea's quarantine policy & $9 /$ Typical \\
\hline & Korea's quarantine maintenance & $4 /$ Variant \\
\hline Macrosystem & Korea's quarantine resources & $10 /$ Typical \\
\hline & Stigma & $16 /$ General \\
\hline & Social Responsibility & $14 /$ Typical \\
\hline
\end{tabular}

\section{Within-Individual: Personality and Belief}

First domain was Within-individual, which contained two categories, personality and belief. Even though all the participants quarantined for the first time and only once, their subjective experiences varied significantly due to their personality and beliefs. Typically, participants reported how their personality impacted their quarantine experience. For example, introverted participants who described themselves as "homebody" were less impacted by the quarantine. These individuals were already having a significant amount of alone time before COVID, quarantine did not clash with their innate tendencies. In fact, one participant said, "I enjoyed the quarantine period because I didn't have to go anywhere". On the contrary, extroverts who normally "feel cramped if I stay at home alone for more than a day" felt "suffocated" because they tend to gain energy from being with others.

Personal beliefs also typically affected the subjective experience of the quarantine. Some participants believed that, if infected, they could be most properly treated in their mother country. They believed that having access to a medical system that they are well-acquainted with was an advantage. Consequently, they chose to come to Korea, making themselves subjects of quarantine. One participant delineated how such belief helped her remain grateful even in the face of the compulsory quarantine. She stated, "As soon as I landed in Korea, I felt less scared because I knew I would be treated well even if I got sick". 
The second domain Microsystem included quarantine space, personal relationships, coresident, student status, and employment status. It was typical for participants to identify their quarantine space as one of the factors that shaped their quarantine experience. One participant noted, "I quarantined at a house with a front yard. I spent a lot of time in the yard, which made my quarantine favorable compared to others". On the other hand, some felt frustrated due to restricted spaces. "I felt suffocated when I looked around the tiny room. I almost had a panic attack because suddenly, I felt so cramped. I questioned how I'm going to survive in this small space".

Also, participants typically reported that connecting with their personal relationships during the quarantine worked against the feeling of isolation. "My grandmother called me every morning to ask, 'How are you? Are you sick?' This was great psychological support". Connecting with others who were also quarantining provided "an unparalleled amount of connectedness". One participant emphasized, "We contacted each other about our own quarantine experience. It was such a great feeling of homogeneity".

Moreover, the existence of a coresident typically altered the participants' quarantine experience. It sometimes functioned as practical aid (e.g., preparing a meal), as well as psychological support. One participant said, "The presence of somebody put me at ease". However, some participants felt hurt when coresidents expressed the anxiety of contagion. One said, "My mom forced me to strictly follow the quarantine rules as if she was a government authority. And she got scared whenever I had to pass through the living room. I ended up telling her that I feel unappreciated".

Last two categories in the Microsystem were related to the social status of individuals, such as student status and employment status. First, although the medium to conduct and evaluate academic performance was changed, nine participants who self-identified as students reported that their selfquarantine revolved around school. One participant reported, "I had to continue my academic duties through remote schooling".

We separated the employment status with student status since one's employment condition variantly impacted the participants' quarantine experience. One self-employed participant recounted, "I had to quarantine in Korea while...there was no one to check on the office space (which was abroad). But I still had to pay the rent. So I was feeling quite anxious and helpless". On the other hand, others continued to work smoothly as they "could do my work on a computer. So my geographic location was not a concern as long as I could work".

\section{Exosystem: Policy, Maintenance, Resources, and Aborad's COVID Measures}

The third domain was Exosystem, as nationwide quarantine measures influenced the subjective experience of quarantine. Korea's quarantine policy, maintenance, and resources, as well as abroad's preventive measures against COVID fell under this domain. First, Korea's quarantine regulations typically dictated the structural aspects of the participants' subjective experience of quarantine. The regulations instructed the participants on how, when, and where to perform all quarantine related tasks. As a result, 
one participant had to "quarantine in a fire-service academy dormitory because quarantining with a coresident (sister) who works closely with a high-risk population (elementary school students) was forbidden".

Secondly, the scrutiny of quarantine adherence monitoring, which differed by the government worker in charge, still generally impacted many aspects of the quarantine experience. One participant had the police on her front door after "forgetting to report my daily temperature and COVID symptoms". Some implied that the monitoring was loose, and that "anyone could fool it" if they were determined to do so. Furthermore, the uncommon experience of having their moves tracked and monitored generated various reactions. Some participants viewed the close monitoring as "troublesome", but more "felt rather protected as...it enables immediate detection of the symptoms...and early response".

In addition, Korea's quarantine resources typically played a significant role in the participants' subjective experience. Number of participants expressed relief in being able to get tested for COVID-19 free of charge, and in being safely escorted to their quarantine space. Quarantine kit was one of the most apparent and tangible resources that influenced the subjective experience. According to the participants, all quarantine kits included essentials such as food supplies, sanitizers, thermometers, garbage bags, and etc. In some cases, the kit included non-essentials too: exercise equipment and a flower pot. Many participants were touched at what their country was doing for them. "It's just protocol, but it was still so touching to receive the aid kit. How caring and thoughtful they are!".

Lastly, participants typically mentioned that the abroad's Exosystem, such as preventive measures against COVID-19, was what drove them to quarantine, as it increased their desire to come to Korea. In participants' residing countries, securing food supplies was difficult, frenzy rumors about martial law were going around, many were dying due to lack of proper medical care, and etc. The unfolding "was so scary... That's why I packed all my things and came to Korea". Many also pointed out that they were likely to receive little support if they actually caught COVID-19 abroad. The difference is captured in the following words: "In Korea, it's like, 'You just abide by our rules, and we will take full care of you. Food, medical service... It's all on us. You just do your job of quarantining'. But there (U.S), it's more like, 'You do everything on your own. You could get infected, so take good care of yourself"'.

\section{Macrosystem: Stigma and Social Responsibility}

Last domain that interacted with participant's quarantine experience was Macrosystem, within which belonged two cultural categories: stigma and social responsibility. First, 10 out of 17 participants typically expressed concerns of being stigmatized. Although all participants were COVID-negative, they feared being labeled as the potential virus-holder. In the beginning of the outbreak, crowds did not hesitate to express rage towards confirmed patients who had unintentionally spread the virus to their communities. Subsequently, the possibility of being stigmatized as a 'spreader' motivated the participants to adhere to the quarantine requirements more. "What if my story got on the news? That was my greatest fear. What if I was broadcasted as a Ms. Something living in Pohang (city in Korea) who went out during quarantine and all of my family was exposed?". Another participant commented, "If my behaviors were 
identified as the cause that the virus spread, everyone would blame and curse me for it. That's why I was even more cautious".

Lastly, participants' social responsibility was another factor that typically enhanced quarantine compliance. They not only wanted to avoid getting reprimanded by their communities, but also wanted to fulfill their responsibility as good standing individuals within their communities. These participants "saw it (quarantine) as something I simply had to do. There was no doubt about it. I have been overseas, and I had symptoms of a cold. It was obvious that I would do this for our country and our people". Some participants were especially considerate of the more vulnerable. For example, one chose to selfquarantine to "make sure to keep my elderly parents safe". Another said, "I work in an academy with young kids. Spreading the virus there would be really terrible. We all know kids' immune systems aren't as strong".

\section{Discussion}

The reported subjective experiences of self-quarantine were not uniformly negative or positive, but rather varied in terms of lifestyle, physical health, psychological experience, and reflection. The findings shed light onto the factors and actions that could maximize adherence to quarantine rules and positive experience of quarantine.

In addition, it was revealed that multiple levels of socioecological variables influence the subjective experience of quarantine: from individual traits, social relations and status, social infrastructure, to culturally shared beliefs. The results inform that interventions on various socioecological levels can improve and worsen the subjective quarantine experiences.

\section{Subjective experience}

One factor that increased quarantine adherence was the participants' infection anxiety. Being categorized as the subjects of self-quarantine increased their perceived likelihood of being infected even though they took the necessary measures to protect themselves from COVID. This increase in perceived possibility of being infected triggered their infection anxiety, causing them to adhere to the quarantine regulations more strictly. In fact, the quarantined experience significantly higher levels of anxiety and depression compared to non-quarantined [15], and anxiety about infection lead to safety actions [16]. Given these findings, following the self-quarantine rules can be interpreted as a safety action against infection anxiety. As this study's findings make it possible to deduce that infection anxiety functioned as a catalyst to strengthen one's quarantine adherence, future research should explore whether or not the infection anxiety persists even after the completion of quarantine.

Additionally, having accurate knowledge about the quarantine protocol was a critical factor that improved adherence to quarantine guidelines. This finding is consistent with the findings of a literature review of 14 studies related to quarantine adherence during infectious disease outbreaks such as Ebola, SARS, and Swine flu, and etc [17]. Consequently, it can be inferred that the strategic and transparent information 
sharing of the Korean government contributed to the high adherence rate of current study's participants. Conversely, a participant who voluntarily started quarantine before the policy was fully in place violated quarantine by leaving the house to get snacks. This echoes the aforementioned review's finding that lack of clear quarantine instruction can cause one to invent their own quarantine rules and acceptable degree of contact with others [17]. In conclusion, current study's findings emphasize the need of assuring the clarity and correctness of self-quarantine guidelines, and of correcting any harmful, false, and misleading information that are being broadcasted [18].

Continuing pre-quarantine lifestyles during quarantine acted as a protective factor for many participants. Quarantine robs people of their freedom to choose what activities to engage in and when, and who to be with. Therefore, being in quarantine can feel like they have no control over their lives. Meanwhile, continuing their pre-quarantine routine enables them to gain a sense of control, as they are able to decide on what to do at which moment. In fact, people's perceived sense of control mediates the effects of positive mental health on stress [19]. Consequently, future research on self-quarantine and sense of control should aim to identify guidelines or activities that could increase people's sense of control in the midst of quarantine.

Generally, self-quarantine is known as an experience that causes psychological distress [20]. Our research findings echo this finding, as participants' psychological experiences included feeling constrained, bored and depressed. Conversely, a new-found phenomenon such as gratitude was also reported. Those who felt gratitude thought highly of Korea's measures against COVID. They decided to come to Korea because they thought that the benefits of coming to Korea overrode the risks of traveling in midst of COVID and the inconvenience of quarantining. They felt safe not only because they were coming to their home country, but also because they could be under Korea's care. In fact, Korea's response to COVID has been heralded as one of the more exemplary models, since the government recognized the need to engage in preventive measures, such as travel restrictions, health screening, quarantine and testing, and public awareness, early on [21]. Subsequently, Korea's response to COVID gave the participants a sense of safety, which resulted in their feelings of gratitude despite being in distressful self-quarantine situations. These results highlight the fact that a country's response to a pandemic has grave implications.

Overall, while this study corroborated previous studies' findings that quarantine leads to psychological difficulties or trauma $[17,20]$, it also shed light into the neutral and positive quarantine experiences such as acceptance, gratitude, and satisfaction. Therefore, it is necessary to acknowledge these positive experiences and conduct various studies on how to maximize the potential protective factors of one's quarantine.

\section{Ecological factors that affect self-quarantine experience}

Unique within-individual factors influenced the self-quarantine experience. As personality refers to unique traits behaving in identical ways across diverse circumstances [22], participants' response to quarantine, a uniformly structured policy, differed because of their distinctive personalities. This is in line with 
findings of a recent study on personality: extraversion, openness, agreeableness, or conscientiousness did not significantly fluctuate during the acute phase of the COVID pandemic [23].

At the same time, a myriad of microsystems such as quarantine space, social status, and relationships constantly shaped the subjective experience. Findings particularly highlight the importance of relationships, even if they are virtual or indirect. Participants appreciated and actively engaged in phone calls and text messaging with friends and family members. As quarantine goes against humans' basic needs of belongings or connectedness [24, 25], alternative fulfilment of such needs could strongly mitigate the psychological threat from being quarantined [26, 27]. To extend this benefit of relational support, policymakers could consider creating an online community for quarantined people to come together to share their experiences, feelings, or tips during and after the quarantine.

Participants' exosystem influenced the subjective experience the most. Although aggressive contact tracing could have raised privacy infringement concerns, many felt protected through their interactions with the Korean government [21]. This can be explained by Korea's 'trace, test, and treat strategy', as this approach traces specifically to enable safe testing and seamless treatment [28]. Resources that existed specifically for the subjects of quarantine also augmented their feelings of being cared for instead of being locked in. However, there is also room for improvement. Since scrutiny of participant monitoring differed by each government official in charge, there was incoherence and confusion regarding the importance of adhering to the rules.

In addition, it is worth noting that the resources that participants identified as conducive to the quarantine experience already existed in Korea before COVID. Affordable and universal health insurance, effective and fast delivery systems, and advanced Internet and communication network services are such resources [29]. Since there is no guarantee that COVID will come to an end, it is imperative for governments to build infrastructures that could ensure convenient and healthy daily lives in the midst of life-threatening pandemic.

Lastly, cultural values such as stigma towards the confirmed patients and social responsibility also played a decisive role in the subjective quarantine experience. In cultures that value collectivism, the self is constructed within a fundamental relatedness to each other [30]. Since South Korea holds collective values such as group harmony and collaboration, becoming the target of stigma is a significant threat to an individual. Moreover, the participants viewed quarantine as an act of taking social responsibility for the community. However, these phenomena cannot be solely attributed to the collective aspect of Korean culture. 'Dataveillant collectivism', ongoing exposure to existential threats and technological development, alongside neo-Confucian traditions, provided a context in which COVID-19 containment was favorable in Korea [31]. Growing literature also acknowledges the interplay of cultural values and jurisdictional responses to the pandemic, social responsibility, and compliance with social distancing and quarantine $[17,32]$. Subsequently, future studies will benefit from taking the complex cultural factors into consideration when exploring how individuals perceive and adhere to quarantine measures. 
Our research findings should be interpreted in light of some limitations. First, our results may reflect the experience of the socio-economically privileged population, thus confining generalization of the results. It is still a meaningful finding given that COVID-19 is equally threatening across all social classes.

Furthermore, lack of external validity is an inherent shortcoming of a qualitative study, as it aims to attain an in-depth understanding of a subgroup of the population rather than to achieve generalization [33]. Nevertheless, underprivileged populations are likely to suffer varying degrees of additional difficulties from quarantine measures. Hence, future studies should expand on the range of samples with respect to socio-economic backgrounds, occupations, and etc.

Secondly, our qualitative approach to quarantine makes it difficult to produce a more computable interpretation of findings. For example, capturing the participants' psychological experiences during quarantine is also possible by utilizing well-established measurements in psychology. Such quantitative approach is also valuable because computing the subjective experience and systematic influences could provide opportunities to empirically verify the effectiveness of individually-used coping strategies and public health policies.

Lastly, although the study contained some practical implications for future quarantine measures, we focused on individual experiences, not the governmental involvements, of quarantines. This was an intentional effort to bring balance to the current literature that has been focusing on governance of COVID-19 at national levels [34,35]. Thus, there was a need to highlight the subjective experience of the individuals who forfeited their freedom for common good. As our findings identify several approaches that governments can take on to improve the individuals' quarantine experience, future studies should closely examine the corresponding results of such public policy modification.

\section{Conclusion}

This study unveils the subjective experience of quarantine implemented as a measure to cope with COVID-19, and the influencing ecological factors of it. Unlike earlier researches that focused on fieldspecific phenomena such as psychology [7], public policy [36], or nutrition [37], our approach was holistic and comprehensive. The findings resonate the need to implement preventive measures against a disease while successfully ensuring the individuals' wellbeing.

\section{Declarations}

Ethics approval and consent to participate: Seoul National University's IRB approved this study. IRB No. 2007/003-026.

Consent for publication: Not applicable.

Availability for data and materials: The datasets used and/or analyzed during the current study are available from the corresponding author on reasonable request. 
Competing interests: The authors declare that they have no competing interests.

\section{Funding: None}

Authors' contributions: GR made substantial contribution to the acquisition of data, interpretation of data, and drafted the work, and substantively revised it. YS made substantial contribution to the design of the work, interpretation of data, and substantively revised it. SM, YM, HP, and SJ equally made substantial contribution to the acquisition of data, interpretation of data, and drafted the work.

All authors read and approved the submitted version of the manuscript. All authors have agreed both to be personally accountable for the author's own contributions and to ensure that questions related to the accuracy or integrity of any part of the work, even ones in which the author was not personally involved, are appropriately investigated, resolved, and the resolution documented in the literature.

Acknowledgements: Not applicable.

\section{References}

1. COVID-19 Public Health Emergency of International Concern (PHEIC) Global research and innovation forum [Internet]. Who.int. 2021 [cited 5 January 2021]. Available from:

https://www.who.int/publications/m/item/covid-19-public-health-emergency-of-internationalconcern-(pheic)-global-research-and-innovation-forum

2. Rubin T. Coronavirus lessons: How South Korea got face masks for everyone and Germany kept death rate down | Trudy Rubin [Internet]. https://www.inquirer.com. 2021 [cited 4 January 2021]. Available from: https://www.inquirer.com/health/coronavirus/coronavirus-masks-shortage-southkorea-germany-fatality-rates-trump-20200407.html

3. Korean Disease Control and Prevention Agency [Internet]. Korean Disease Control and Prevention Agency. 2021 [cited 3 February 2021]. Available from: http://www.kdca.go.kr/gallery.es?

$\mathrm{mid}=\mathrm{a} 20503020000 \& \mathrm{bid}=0003 \&$ act $=$ view $\&$ list_no $=144811$

4. Choi Y. The Power of Collaborative Governance: The Case of South Korea Responding to COVID-19 Pandemic. World Medical \& Health Policy. 2020;12(4):430-442.

5. Brooks S, Webster R, Smith L, Woodland L, Wessely S, Greenberg N et al. The psychological impact of quarantine and how to reduce it: rapid review of the evidence. The Lancet. 2020;395(10227):912920.

6. Chen D, Song F, Tang L, Zhang H, Shao J, Qiu R et al. Quarantine experience of close contacts of COVID-19 patients in China: A qualitative descriptive study. General Hospital Psychiatry. 2020;66:8188.

7. Saurabh K, Ranjan S. Compliance and Psychological Impact of Quarantine in Children and Adolescents due to Covid-19 Pandemic. The Indian Journal of Pediatrics. 2020;87(7):532-536. 
8. Sidor A, Rzymski P. Dietary Choices and Habits during COVID-19 Lockdown: Experience from Poland. Nutrients. 2020;12(6):1657.

9. Mattioli A, Sciomer S, Cocchi C, Maffei S, Gallina S. Quarantine during COVID-19 outbreak: Changes in diet and physical activity increase the risk of cardiovascular disease. Nutrition, Metabolism and Cardiovascular Diseases. 2020;30(9):1409-1417.

10. Bronfenbrenner U. Ecology of Human Development: Experiments by Nature and Design. Harvard University Press; 1979.

11. Hill C, Thompson B, Williams E. A Guide to Conducting Consensual Qualitative Research. The Counseling Psychologist. 1997;25(4):517-572.

12. Hill C, Knox S, Thompson B, Williams E, Hess S, Ladany N. Consensual qualitative research: An update. Journal of Counseling Psychology. 2005;52(2):196-205.

13. Hill C. Consensual qualitative research. Washington, DC: American Psychological Assoc.; 2012.

14. Thompson BJ, Vivino BL, Hill CE. Coding the data: Domains and core ideas. In Hill CE, editor. Consensual qualitative research: A practical resource for investigating social science phenomena. American Psychological Association; 2011: p. 103-116.

15. Tang F, Liang J, Zhang H, Kelifa M, He Q, Wang P. COVID-19 related depression and anxiety among quarantined respondents. Psychology \& Health. 2020;36(2):164-178.

16. Reiss S, Franchina V, Jutzi C, Willardt R, Jonas E. From anxiety to action-Experience of threat, emotional states, reactance, and action preferences in the early days of COVID-19 self-isolation in Germany and Austria. PLOS ONE. 2020;15(12):e0243193.

17. Webster R, Brooks S, Smith L, Woodland L, Wessely S, Rubin G. How to improve adherence with quarantine: rapid review of the evidence. Public Health. 2020;182:163-169.

18. Transparency, communication and trust: The role of public communication in responding to the wave of disinformation about the new Coronavirus [Internet]. OECD. 2021 [cited 21 February 2021]. Available from: https://www.oecd.org/coronavirus/policy-responses/transparency\%20communication\%20-and-trust-bef7ad6e/

19. Brailovskaia J, Margraf J. Predicting adaptive and maladaptive responses to the Coronavirus (COVID-19) outbreak: A prospective longitudinal study. International Journal of Clinical and Health Psychology. 2020;20(3):183-191.

20. Serafini G, Parmigiani B, Amerio A, Aguglia A, Sher L, Amore M. The psychological impact of COVID19 on the mental health in the general population. QJM: An International Journal of Medicine. 2020;113(8):531-537.

21. Kim M, Cho W, Choi H, Hur J. Assessing the South Korean Model of Emergency Management during the COVID-19 Pandemic. Asian Studies Review. 2020;44(4):567-578.

22. Roberts B. Back to the future: Personality and Assessment and personality development. Journal of Research in Personality. 2009;43(2):137-145. 
23. Sutin A, Luchetti M, Aschwanden D, Lee J, Sesker A, Strickhouser J et al. Change in five-factor model personality traits during the acute phase of the coronavirus pandemic. PLOS ONE. 2020;15(8):e0237056.

24. Maslow A. A theory of human motivation. Psychological Review. 1943;50(4):370-396.

25. Frey L, Wilhite K. Our Five Basic Needs. Intervention in School and Clinic. 2005;40(3):156-160.

26. Hagerty S, Williams L. The impact of COVID-19 on mental health: The interactive roles of brain biotypes and human connection. Brain, Behavior, \& Immunity - Health. 2020;5:100078.

27. Kuwahara K, Kuroda A, Fukuda Y. COVID-19: Active measures to support community-dwelling older adults. Travel Medicine and Infectious Disease. 2020;36:101638.

28. Tackling COVID-19 Health, Quarantine and Economic Measures: Korean Experience [Internet]. 2020 [cited 5 January 2021]. Available from: https://ecck.or.kr/wp-content/uploads/2020/03/TacklingCOVID-19-Health-Quarantine-and-Economic-Measures-of-South-Korea.pdf

29. Shin Y, Lee J. South Korea's proactive approach to the COVID-19 global crisis. Psychological Trauma: Theory, Research, Practice, and Policy. 2020;12(5):475-477.

30. Markus H, Kitayama S. Culture and the self: Implications for cognition, emotion, and motivation. Psychological Review. 1991;98(2):224-253.

31. 31. Kasdan D, Campbell J. Dataveillant Collectivism and the Coronavirus in Korea: Values, Biases, and Socio-Cultural Foundations of Containment Efforts. Administrative Theory \& Praxis. 2020;42(4):604-613.

32. Velamoor V, Persad E. Covid-19: Cultural perspectives. Asian Journal of Psychiatry. 2020;53:102439.

33. Morrow S. Qualitative Research in Counseling Psychology. The Counseling Psychologist. 2007;35(2):209-235.

34. Shah J, Shah J, Shah J. Quarantine, isolation and lockdown: in context of COVID-19. Journal of Patan Academy of Health Sciences. 2020;7(1):48-57.

35. Moon M. Fighting COVID -19 with Agility, Transparency, and Participation: Wicked Policy Problems and New Governance Challenges. Public Administration Review. 2020;80(4):651-656.

36. Anderson M, Mckee M, Mossialos E. Developing a sustainable exit strategy for COVID-19: health, economic and public policy implications. Journal of the Royal Society of Medicine. 2020;113(5):176178.

37. Muscogiuri G, Barrea L, Savastano S, Colao A. Nutritional recommendations for CoVID-19 quarantine. European Journal of Clinical Nutrition. 2020;74(6):850-851.

\section{Supplementary Files}

This is a list of supplementary files associated with this preprint. Click to download.

- Appendixglobalizationandhealth.docx 